\title{
On ARQ Scheme With Adaptive Error Control
}

\author{
Hlaing Minn, Student Member, IEEE, Mao Zeng, and Vijay K. Bhargava, Fellow, IEEE
}

\begin{abstract}
Adaptive automatic repeat request (ARQ) schemes are quite effective for throughput enhancement in time-varying mobile channel environments. In this paper, an ARQ scheme with adaptive error-correcting codes is considered. A robust channel sensing algorithm by making use of XOR-ing is proposed for link adaptation. With the proposed channel estimation algorithm, an adaptive scheme that adapts its error correcting codes according to channel conditions is presented. The efficiency of the proposed adaptive ARQ scheme is evaluated in terms of throughput performance by computer simulation for a time-varying mobile channel characterized by Rayleigh fading and log-normal shadowing. As an enhancement in counteracting long deep fades, the incorporation of frequency diversity into adaptive ARQ scheme is also investigated and the simulation results show that this scheme is quite efficient in counteracting long deep fades.
\end{abstract}

Index Terms-Adaptive error control, adaptive automatic repeat request (ARQ), adaptive $F H$, channel sensing, frequency hopping (FH), time-varying mobile radio channel.

\section{INTRODUCTION}

A UTOMATIC repeat request (ARQ) and hybrid ARQ error control strategies are usually incorporated in the system design for nondelay-sensitive communications services with very high system performance requirement. If those services are provided through mobile wireless radio channel, the nature of the time-varying multipath fading channel has to be taken into consideration in the system design. A practical mobile radio channel induces a time-varying response with bursty errors due to multipath fading and shadowing effects. During the fades the channel becomes too noisy and at the other times it well-behaves. For such a time-varying channel, it is clear that the use of a single error control strategy will not yield the optimal throughput. Therefore, in order to counteract the fading channel conditions, adaptive schemes need to be incorporated into ARQ schemes, e.g., the use of different error control strategies for different channel conditions needs to be established for an ARQ system with adaptive error-correcting codes. Generally, adaptive scheme can be performed not only on error-control code, but also on packet length, modulation format, etc.

Recently, there has been considerable interest in adaptive ARQ schemes [1]-[8]. The basic idea is by dynamically

Manuscript received April 6, 2000; revised January 3, 2001. This work was supported in part by the Natural Sciences and Engineering Research Council (NSERC) of Canada under a Strategic Project Grant and in part by Telus Mobility, Cellular.

H. Minn and V. K. Bhargava are with the Department of Electrical and Computer Engineering, University of Victoria, Victoria, BC V8W 3P6, Canada (e-mail: hminn@ece.uvic.ca; bhargava@ece.uvic.ca).

M. Zeng is with Motorola, Atlanta, GA 30328 USA.

Publisher Item Identifier S 0018-9545(01)09856-5. changing the operation mode according to channel state information, the higher throughput is realized over a wide range of channel conditions. For real-time implementation, the channel state information needs to be estimated reliably and effectively. That means that in order to track channel variations closely, reliable estimation of the channel state information should be carried out within short enough intervals (which may involve accurate estimation of the signal strength via signal power measurement or with pilot tone transmission, and, in turn, give rise to high implementation complexity). Recently, indirect approaches [1]-[3], [6], [9], [10] for channel state estimation have been proposed in the literature.

In [1], Hara et al. estimated the packet error rate by using the count of negative acknowledgement (NACK - not sucessful) packets in a fixed observation interval. However, the choice of observation interval for reliable estimation highly depends on the fading/shadowing condition. If the observation interval is small relative to the channel fading rate, reliable estimate of channel state information cannot be achieved. Consequently, the mode change decision based on the unreliable channel estimate may lose significant potential throughput gain achievable by an adaptive scheme. On the other hand, if the observation interval is large relative to the channel fading rate, the potential throughput gain may be lost significantly due to the delay in responding to the channel state changes. Moreover, the channel states at different modes of the adaptive system would not be the same and hence the use of one fixed observation interval may not be of wise choice. Using a fixed observation interval for channel state estimation in an unknown fading environment gives an estimate averaged over the interval but does not give much information on how likely the next packet(s) will encounter similar channel conditions.

In [2], packet error rate was estimated using count of NACK packets in different observation intervals for different modes of the adaptive scheme. The Markov channel model with known state transition probabilities and known channel transition probabilities within each state is assumed. Based on the assumed channel model and channel statistics, the observation intervals are found. However, this approach might not be applicable to an unknown fading environment. The applicability of the design parameters obtained with assumed channel model and channel statistics to the practical time-varying fading channel needs further investigation. Using different observation intervals for different modes of the adaptive scheme may be conceptually viewed as a way to achieve different channel state estimates in different modes with the same estimation reliability. It tries to avoid unnecessarily long observation intervals for some modes and insufficient observation intervals for others. For the known channel, the fading rate information is incorporated in the design by the known channel statistics. However, in an unknown 
fading environment, it does not give much information on how likely the next packet(s) will experience similar channel conditions.

In [3], a sequential scheme for channel state estimation was proposed where each transmitted packet is scored based on the outcome of the decoding process [i.e., acknowledgement (ACK-sucessful) or NACK]. When the cumulative score crosses a decision threshold, the coding strategy (mode) is changed and the sequential inspection scheme is restored using the same scoring routine with different weighting and decision threshold constants. The Markov channel model with known state transition probabilities and known channel transition probabilities within each state is assumed. Based on the known channel statistics, the weighting and decision threshold constants for each mode is found by optimizing the throughput using reliability as a constraint. For an unknown fading environment, the approach might not be applicable. The applicability of the design parameters obtained with assumed channel model and channel statistics to the practical time-varying fading channel needs further investigation.

In [4], XOR-ing of the two consecutive erroneous copies is used to estimate the channel bit error rate (BER) for an adaptive ARQ scheme with no error correction in a stationary channel. As originally stated, XOR-ing was first used by Sindhu in [5] to locate the errors. But how to deal with the case when there is no consecutive unsuccessful packets was not considered by [4]. Although [4] states that their channel BER estimation can be applied to any adaptive scheme, we notice that for ARQ schemes with adaptive error correction codes the channel BER may not be estimated properly due to error correction of the forward error correction (FEC) code. Specifically, their method gives channel BER estimation for one direction only (i.e., from the good channel state to the bad channel state direction). In this paper, how to estimate channel error rate for the other direction (i.e., the from bad channel state to the good channel state) is also addressed.

In [6], the count of successive ACK or NACK is used for mode change decision in a two-mode adaptive system in a stationary channel. If $\alpha$ successive ACKs are received in mode-H (high error rate mode), the adaptive system assumes that the channel is returning to good state and changes its mode to mode-L (low error rate mode). If $\beta$ successive NACKs are received in mode- $\mathrm{L}$, the adaptive system assumes that the channel is deteriorating into a bad state and changes its mode to mode- $\mathrm{H}$. The values of the design parameters $\alpha$ and $\beta$ are found by trials. A way of finding adaptive system parameters is proposed in [9], [10] where the parameters of the adaptive ARQ system with three modes are found by optimization of the throughput assuming a stationary channel.

The use of successive ACKs or NACKs gives some information on how likely the next packet(s) will experience similar channel conditions. However, finding the optimum parameter values of successive ACKs or NACKs in a time-varying fading channel is an unfeasible task. Finding the adaptive system parameters by optimization of the throughput assuming a stationary channel is one way to get suitable design parameters. Although the designed adaptive system parameters do not represent the optimal ones for a practical time-varying fading channel due to the stationary channel assumption, their applicability to a practical time-varying fading channel has been confirmed by simulation studies in [10]. However, for an adaptive system with more than three modes, the task involved in this approach becomes tedious. In this paper, we present an alternative approach for ARQ scheme with adaptive error control codes, particularly using the codes with known error correction capability such as Reed-Solomon codes. The proposed approach avoids the throughput calculation and optimization in finding the adaptive system design parameters used in [9], [10]. It uses the count of consecutive ACKs or NACKs just to determine whether the selection of the most suitable mode should be performed or not; the operation mode may not necessarily be changed, in contrast to previous use of consecutive ACKs or NACKs count to definitely change the adaptive system mode. Based on the proposed channel error estimate together with the error-correction capabilities of the FEC codes used, the decision on the mode in which the system should operate is made. The mode change may not be necessary for some channel conditions.

For circumstances of deep fades in slow fading environment, even stronger FEC code may not be helpful in achieving throughput gain. For such situation, some other dimension diversity schemes, such as frequency dimension, may be incorporated to improve the system performance. In this paper, an adaptive ARQ scheme incorporated with frequency hopping (FH) concept is also investigated.

The paper is organized as follows. In Section II, the adaptive ARQ scheme based on the throughput optimization with stationary channel assumption [9], [10] is briefly described. In Section III, an ARQ scheme with adaptive error control is introduced, and a robust channel sensing algorithm is also proposed for the link adaptation. In Section IV, the introduction of the FH concept into adaptive ARQ scheme is discussed. The simulation study of the proposed adaptive ARQ schemes are presented in Section V. The results of [10] and random nonadaptive FH system are also included for comparison. Finally, conclusions are given in Section VI.

\section{ADAPTIVE ARQ SCHEME}

The general description of adaptive ARQ scheme with $J$ modes is given in Fig. 1. Based on the channel estimation in each mode, the most suitable mode for the next transmission is chosen and the system is switched to the chosen mode. As an example, the adaptive ARQ scheme proposed in [9], [10] is briefly described in the following where mode change is allowed only to adjacent modes and channel estimation is based on the count of consecutive ACKs or consecutive NACKs. The corresponding Markov chain representation of the adaptive ARQ system with three modes (namely, mode $\mathrm{L}, \mathrm{M}$, and $\mathrm{H}$ ) is shown in Fig. 2. While operating in mode L, if $\alpha$ consecutive NACKs are received, the adaptive system is switched to mode $\mathrm{M}$. While in mode $\mathrm{M}$, if $\beta$ consecutive ACKs are received, the adaptive system is switched back to mode $\mathrm{L}$; on the other hand, if $\gamma$ consecutive NACKs are received, the adaptive system is switched to mode $\mathrm{H}$. If $\lambda$ consecutive ACKs are received while operating in mode $\mathrm{H}$, the adaptive 


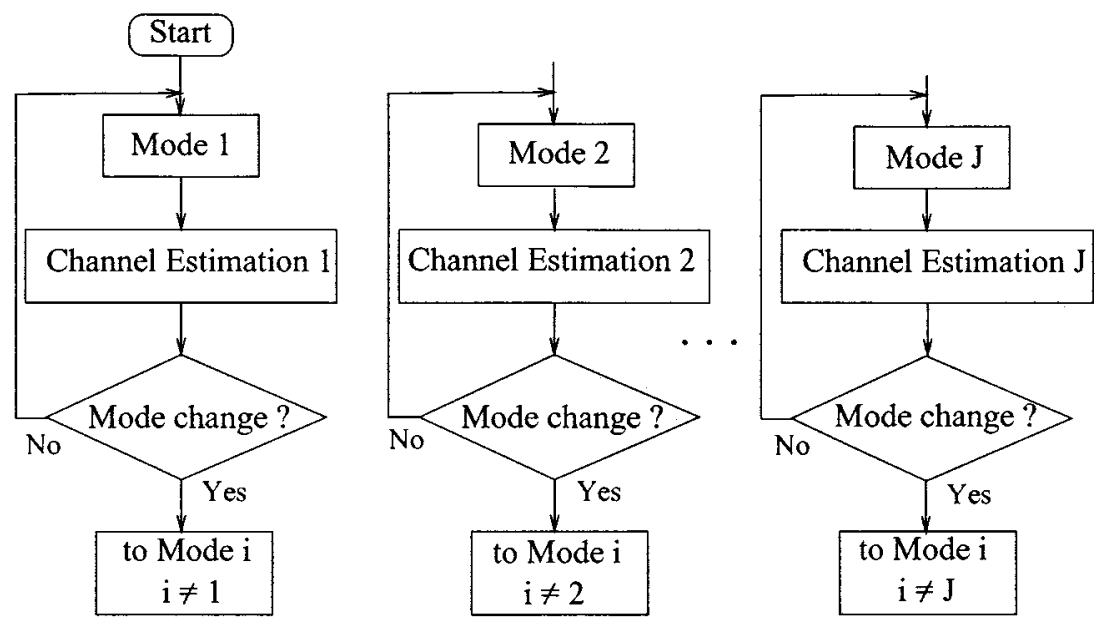

Fig. 1. General description of adaptive ARQ scheme with $J$ modes.

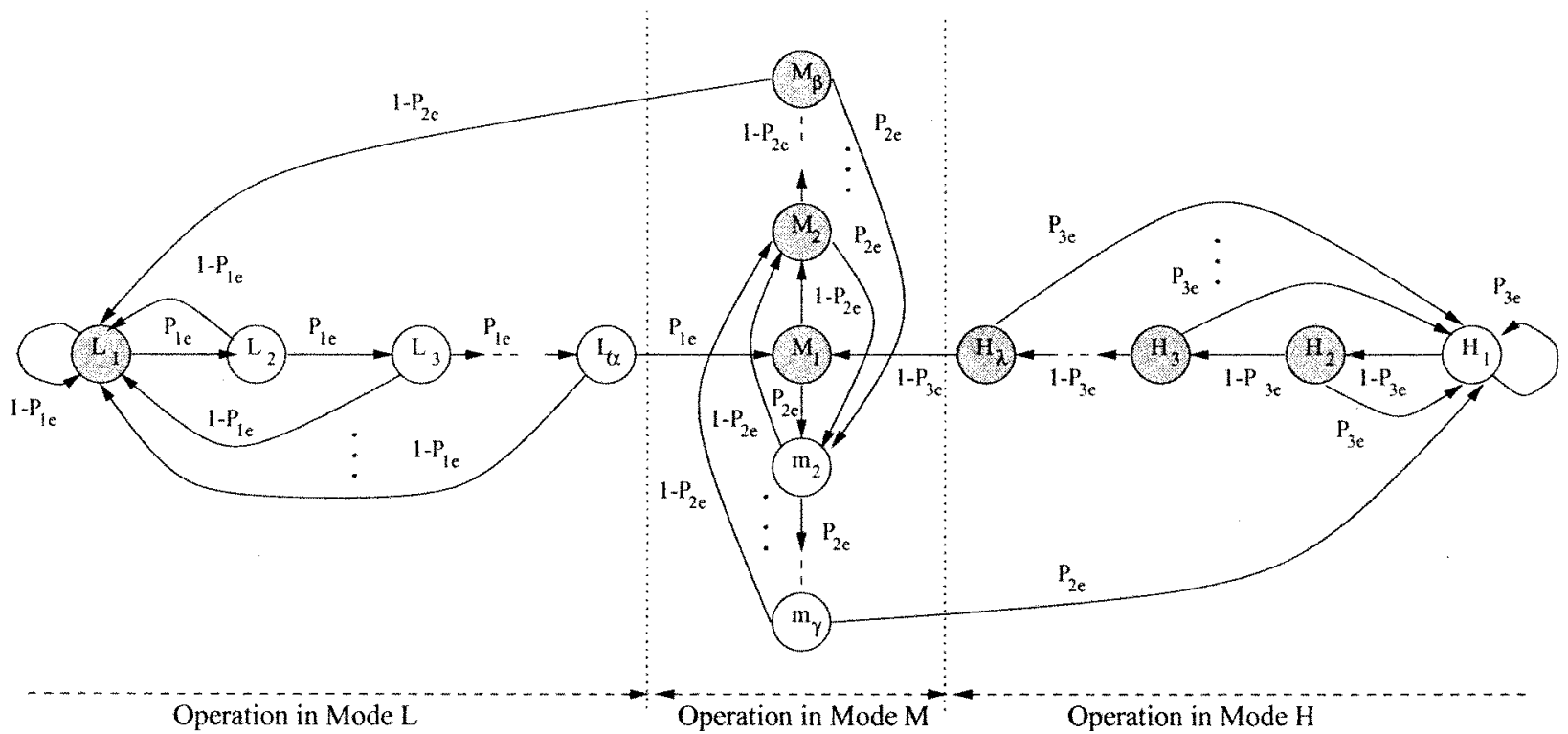

Fig. 2. Markov chain representation of adaptive ARQ scheme with three modes based on stationary channel assumption.

system is switched back to mode M. Assuming a stationary channel, the adaptive system parameters $(\alpha, \beta, \gamma, \lambda)$ are found by optimization of the throughput efficiency over a range of symbol error probabilities. The throughput efficiency of the adaptive ARQ scheme is given by

$$
\eta=\frac{K}{T}
$$

where $T$ is the average number of bits that would have been transmitted for a successful reception of a packet of $K$ information bits and is given by

$$
T=p_{L_{1}} T_{L_{1}}+p_{M_{1} H} T_{M_{1}}+\sum_{i=2}^{\beta} p_{M_{i}} T_{M_{i}}+\sum_{j=2}^{\lambda} p_{H_{j}} T_{H_{j}}
$$

where

$$
\begin{aligned}
p_{X} & =\frac{P_{X}}{P_{\text {new }}} \\
P_{\text {new }} & =P_{L_{1}}+P_{M_{1 H}}+\sum_{i=2}^{\beta} P_{M_{i}}+\sum_{j=2}^{\lambda} P_{H_{j}}
\end{aligned}
$$

and

$$
P_{M_{1 H}}=P_{M_{1}}-P_{L_{1}} P_{1 e}^{\alpha} .
$$

In the above equation, $T_{x}$ stands for the average number of bits that would have been transmitted for a successful reception of a packet of $K$ information bits whose first transmission is initiated in state $x, P_{x}$ is the steady-state probability of state $x, P_{\text {new }}$ is the steady-state probability of a new packet to be transmitted, $p_{x}$ is the conditional probability of a new packet initiated in state $x$ given that a new packet is to be transmitted.

\section{Proposed Adaptive ARQ Scheme}

\section{A. Channel Error Rate Estimation}

In [4], XOR-ing (originally used by [5]) of the two consecutive errorneous copies is used to estimate the channel BER for an adaptive ARQ scheme with no error correction in a stationary channel. The work in [4] did not address the case with error-correcting code in a typical time-varying mobile channel environment. If the method of [4] is directly applied to the case 


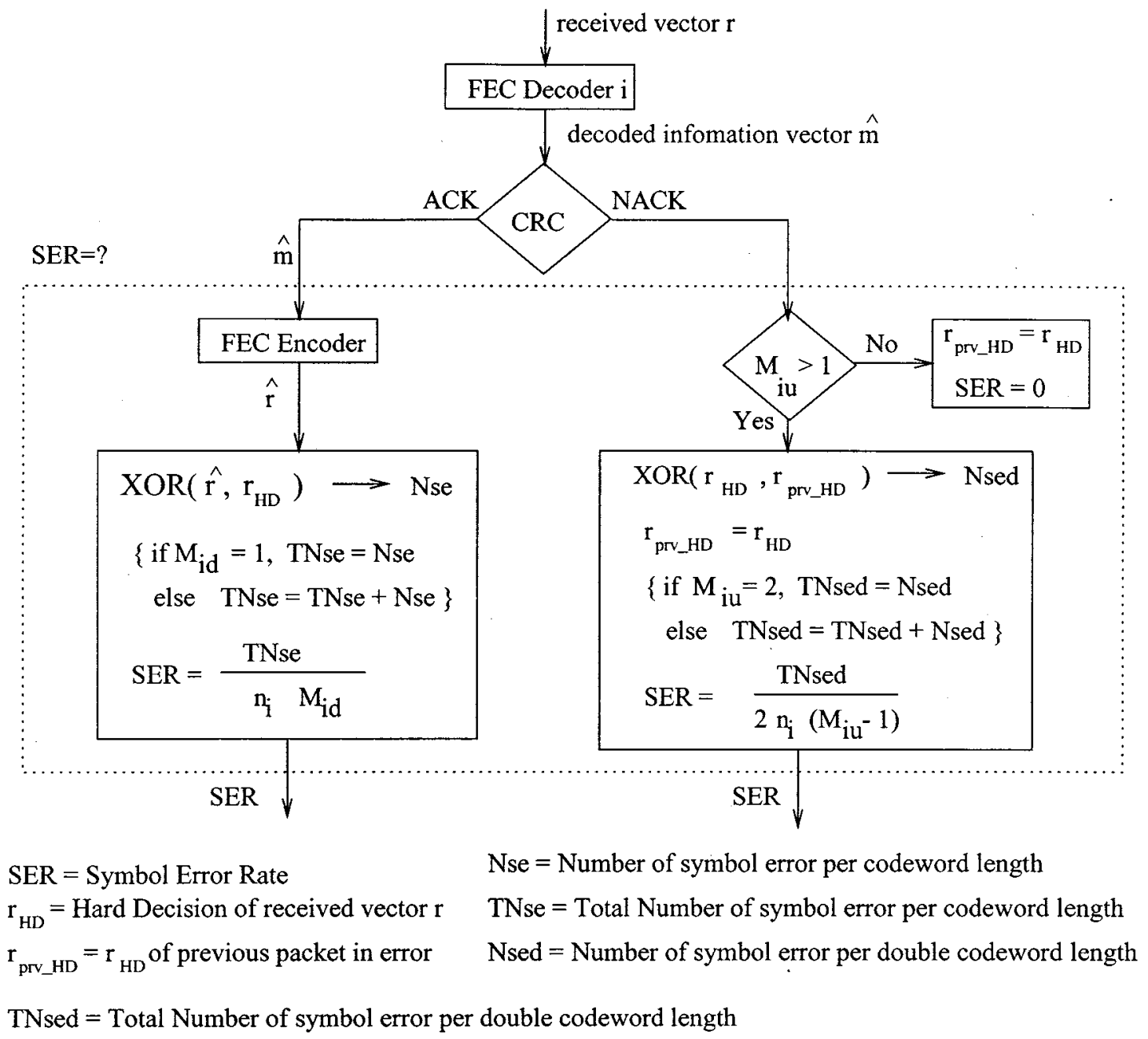

Fig. 3. Proposed channel error rate estimation.

with error-correcting code in time-varying mobile channel, the channel BER may not be estimated properly due to error correction of the FEC code. Specifically, their method gives channel BER estimation for one direction only (i.e., from a good channel state to a bad channel state direction). In this paper, we address channel error rate estimation in a typical time-varying mobile channel with error-correcting code of nonbinary alphabet (the binary alphabet case is just a simplified case of nonbinary one). We describe how to estimate channel error rate for both directions (i.e., from the bad channel state to the good channel state and vice versa).

The proposed channel error rate estimation scheme is given in Fig. 3. Error-correcting codes with nonbinary symbols are considered. Mode 1 uses the code with the least error correction capability (usually no error correction capability), the larger mode number indicates the code with stronger error correcting capability and mode $J$ uses the code with the strongest error correcting capability among all $J$ modes. While operating in mode $i$, the count of successive NACKs is stored in $M_{i u}$, and the count of successive ACKs is stored in $M_{i d}$. Although FEC can do error detection, for simplicity we assume, without loss of generality, there is a cyclic redundancy check (CRC) for error detection on top of FEC for error correction. Suppose that after
FEC decoding, a packet error is detected by CRC. If the previous packet is received successfully (then $M_{i u}=1$ ), the hard decisions (HD) of the current erroneous packet is stored for future use in channel error rate estimation. If the previous packet is also erroneous (then $M_{i u}=2$ ), the HDs of the current and previous erroneous packets are XOR-ed to estimate the bit errors in a combined double packet length interval. Any estimated bit error(s) within a symbol causes an estimated symbol error. The estimated bit errors are transformed to estimated symbol errors. Then, the estimate of number of symbol error per double codeword length $N$ sed is obtained by averaging over all codewords of the packet. The successive estimates of the number of symbol error per double codeword length are accumulated in $T N$ sed. Then the symbol error rate (SER) is estimated by

$$
\mathrm{SER}=\frac{T N \text { sed }}{2 n_{i}\left(M_{i u}-1\right)}
$$

where $n_{i}$ is the number of symbols per codeword in mode $i$. Suppose that after FEC decoding, there be no error detected by CRC. Then the estimate of transmitted packet $\hat{r}$ is obtained by FEC encoding the successfully decoded message and XOR-ed with the HD of the received packet to obtain the estimate of bit errors in the successful packet. Next, the estimated bit errors are 


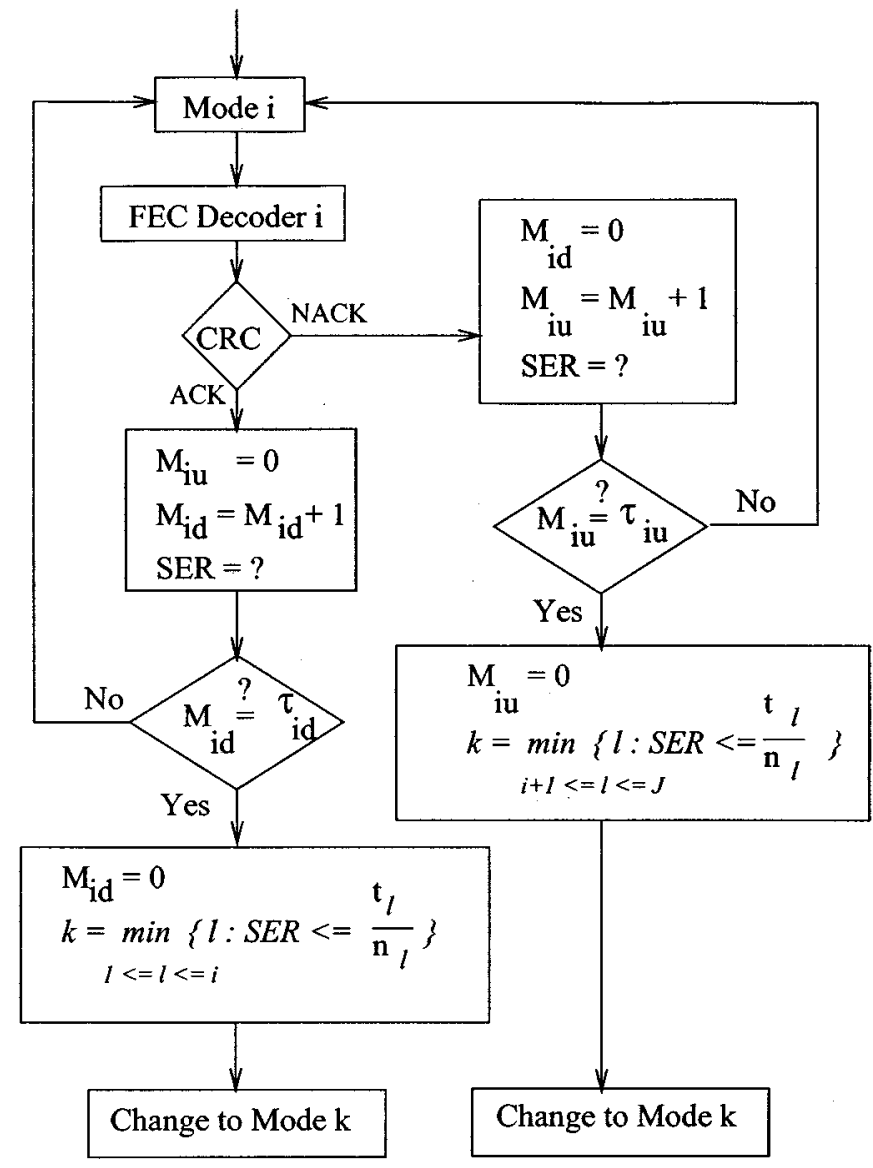

Fig. 4. Proposed adaptive ARQ scheme operating in mode $i, 2 \leq i \leq J-1$.

transformed to estimated symbol errors in the packet. Then the estimate of number of symbol error per codeword length $\mathrm{Nse}$ is obtained by averaging over all codewords of the packet. The successive estimates of $N$ se are accumulated in $T N s e$ and the SER estimate is given by

$$
\mathrm{SER}=\frac{T N s e}{n_{i} M_{i d}} .
$$

Note that $T N$ se and $T N$ sed can be stored in the same memory since only one is required at a time.

\section{B. Proposed Adaptive Scheme}

Consider an adaptive ARQ scheme with $J$ modes. The operation of proposed adaptive ARQ scheme in mode $i$, where $2 \leq i \leq J-1$ is shown in Fig. 4 and the operations in mode 1 and mode $J$ are shown in Fig. 5. While operating in mode $i$, the receiver decides which mode is the most suitable one for the next transmission

1) after $\tau_{i d}$ th continuous successful packet at the receiver (i.e., when $M_{i d}=\tau_{i d}$ ) or

2) after $\tau_{i u}$ th continuous unsuccessful packet at the receiver (i.e., when $M_{i u}=\tau_{i u}$ ).

In the first case, the $\tau_{i d}$ th successive ACK packet on the feedback channel also contains the information on which mode the next packet is to be transmitted. In the second case, the $\tau_{i u}$ th successive NACK packet on the feedback channel also contains the information on which mode the next packet is to be transmitted. In other feedback transmissions, packet does not contain the mode number information.

The most suitable mode for the next transmission is chosen as follows. When the successive ACKs in mode $i$ trigger the mode selection procedure, the symbol error rate obtained by the channel error rate estimation is compared against that defined by the error correction capabilities of modes 1 to $i$ (i.e., $\left.t_{1}, t_{2}, \ldots, t_{i}\right)$, and the most suitable mode $k$ for the next transmission is chosen according to the following:

$$
k=\min _{1 \leq l \leq i}\left\{l: \mathrm{SER} \leq \frac{t_{l}}{n_{l}}\right\} .
$$

When the successive NACKs in mode $i$ trigger the mode selection procedure, the SER obtained by the channel error rate estimation is compared against that defined by the error correction capabilities of modes $i+1$ to $J$ (i.e., $t_{i+1}, t_{i+2}, \ldots, t_{J}$ ), and the most suitable mode $k$ for the next transmission is chosen according to the following:

$$
k=\min _{i+1 \leq l \leq J}\left\{l: \mathrm{SER} \leq \frac{t_{l}}{n_{l}}\right\} .
$$

The proposed adaptive ARQ scheme described above is discussed in a general sense with the parameters $\left\{\tau_{i d}, \tau_{i u}\right\}$. To find the optimal parameters $\left\{\tau_{i d}, \tau_{i u}\right\}$ for a practical time-varying multipath fading channel is analytically unfeasible. Our choice for these parameters is $\tau_{i d}=\tau_{i u}=2\left(\tau_{1 d}\right.$ and $\tau_{J u}$ are not required). The choice is based on the reasoning that it is much likely for the next packet (new/old) to encounter similar channel condition after two successive packet successes/failures in a slow fading environment. The channel condition for the next packet is hence estimated based on the two successive packets of success/failure. Then the resulting channel error rate estimate for the next packet and the error correction capabilities of the modes are used to choose the most suitable mode for the next packet transmission.

Some differences between the adaptive ARQ schemes described in Sections II and III are discussed in the following. The scheme of Section II requires calculation of throughput efficiency expression and optimization of the throughput to find the adaptive system parameters. These parameters are used to determine mode changes. The channel error rate is indirectly estimated by these parameters. The scheme of Section III uses fixed adaptive system parameters. These parameters are used for triggering the mode selection procedure not for the mode change. The channel error rate is estimated by the XOR-ing method over successive packets the number of which is determined by these parameters. And the error correction capabilities of the operation modes are also used in mode selection.

\section{Proposed Frequency-Hopped AdAPtive ARQ Scheme}

For circumstances of deep fades in slow fading environment, even stronger FEC code may not be helpful in achieving throughput gain. For example, in very deep fades with long duration, trying to send packets with stronger FEC codes may be useless and power dissipating. For such situations, incorporation of FH concept into adaptive ARQ scheme is also investigated in this paper. Usually, shadowing effects are 


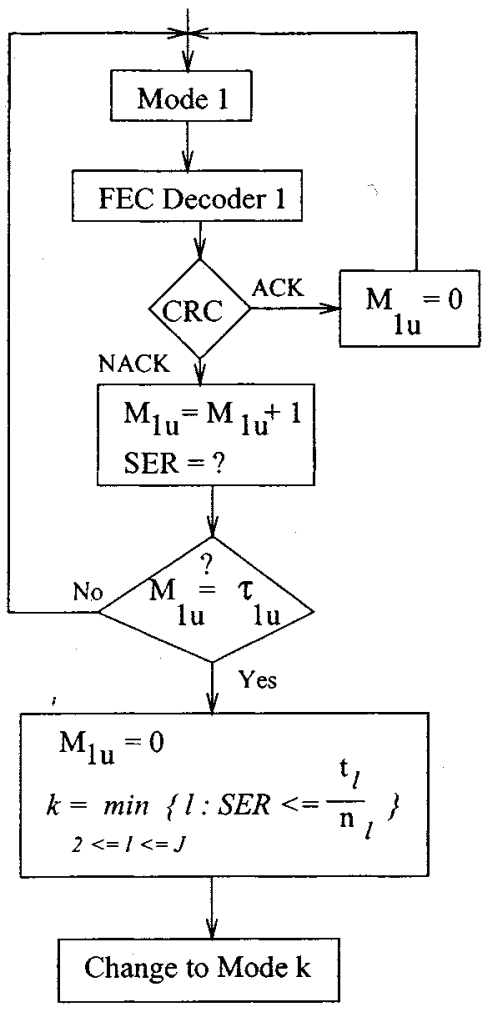

Fig. 5. Proposed adaptive ARQ scheme operating in mode 1 and $J$.

caused by terrain environment, hopping the channel frequency into another one may not cure the shadow fading. However, multipath fading in a slow fading environment can be counteracted by FH since it depends on the carrier frequency.

In conventional FH systems, the carrier frequency is hopped at a fixed rate. In contrast, our proposed frequency-hopped adaptive ARQ scheme (FH-adaptive ARQ) hops the carrier frequency only when it is necessary. For an adaptive ARQ system with $J$ modes, when $\tau_{J u}$ successive packet failures are detected while operating in mode $J, \mathrm{FH}$ is initiated. The required signaling including the new carrier frequency information may be communicated on a dedicated control channel with sufficient error protection. On completion of required signaling, the adaptive system starts to operate with the new carrier frequency in the mode $J$. Since the packet which is transmitted on a new carrier frequency has already experienced a certain amount of delay, we choose mode $J$ for the system to start operating on new carrier frequency, taking into account the aspect of delay. If the system has no reserved carrier frequencies for $\mathrm{FH}$ purpose but is designed to allow $\mathrm{FH}$ to another free user carrier frequency, then the same carrier frequency will be reused for fully loaded instances. In that situation, FH procedure is performed but actual $\mathrm{FH}$ is not accomplished.

Next, the choice of $\tau_{J u}$ value is a tradeoff between counteracting to deep fades and signaling load on the system. The choice of small value, say $\tau_{J u}=1$, would more likely counteract deep fades by means of frequency diversity of FH but, on the other hand, the more often requirement of signaling would burden the system. The choice of large value would reduce the signaling load on the system but would have less chance of utilizing frequency diversity effect of $\mathrm{FH}$, hence possibly re-



ducing the throughput improvement. In our simulation study, the performance of FH-adaptive ARQ for different choice of FH threshold parameter value $\tau_{J u}$ will also be studied.

\section{Performance Evaluation by Simulation}

\section{A. System Parameters of Simulation Study}

The exact analytical calculation of throughput efficiency of adaptive ARQ schemes in a practical time-varying mobile radio channel is an intractable task and hence, the simulation approach is adopted here to evaluate the performance of adaptive ARQ schemes in a typical mobile radio channel. Adaptive stop-and-wait (SW) ARQ schemes with three error-control codes are considered in our simulation study. Usually packet length is designed based on the application type, channel environment, and performance requirement. In our simulation study of adaptive ARQ schemes, without significantly affecting the adaptive schemes' performance comparison, we simply assume that one data packet contains only one codeword. We also assume that packets contain only information bits neglecting the synchronization and control bits. All the codes of different modes contain the same length of information bits. In mode 1 , which corresponds to low error rate mode mode- $\mathrm{L}$ in [9], [10], only CRC is used for error detection. Besides CRC, in modes 2 and 3, which correspond to moderate error rate mode node- $\mathrm{M}$ and high error rate mode mode- $\mathrm{H}$, respectively, in [9], [10], a punctured Reed-Solomon code RS $(21,15)$ and a Reed-Solomon code RS $(31,15)$ are used for error correction, respectively. Binary phase-shift keying (BPSK) modulation with coherent detection is considered. 
The throughput efficiencies of the adaptive ARQ scheme with stationary channel assumption, the proposed adaptive ARQ scheme, and nonadaptive ARQ schemes using each code of adaptive ARQ schemes mentioned above are compared for the mobile speeds of 1,10 , and $50 \mathrm{~km} / \mathrm{h}$ and long-term signal-to-noise ratio (SNR) range of 5 to $25 \mathrm{~dB}$. The long-term SNR is defined based on the long interval of $2^{19}$ samples (i.e., approximately $36.41 \mathrm{~s}$ at $14.4 \mathrm{kHz}$ ). The carrier frequency of $900 \mathrm{MHz}$ and data rate of $14.4 \mathrm{~kb} / \mathrm{s}$ are used. The round-trip delay of two Reed-Solomon symbol intervals is assumed. In throughput evaluation, perfect error detection of CRC is assumed and the CRC parity bits are not included in throughput calculation. The perfect feedback channel is assumed. The parameters of the adaptive ARQ scheme with stationary channel assumption are $(\alpha, \beta, \gamma, \lambda)=(1,5,2,5)$ [9]. For the proposed scheme, the round-trip delay associated with the occasions of ACK/NACK containing mode number information is larger than the other occasions of normal ACK/NACK, and for illustration, is assumed three Reed-Solomon symbol interval. ARQ scheme which includes the FH concept when required, is also investigated in the same environment. For FH-adaptive ARQ scheme, the FH setup time, comprised of the signaling (handshaking) time and round-trip delay for a complete FH, is assumed for illustration as one packet length in mode 3 plus two normal round-trip delays.

As a comparison to the proposed FH-adaptive ARQ scheme, nonadaptive $\mathrm{FH}$ system is also evaluated. Three cases of nonadaptive FH system with CRC, CRC $+\mathrm{RS}(21,15)$ and CRC + RS $(31,15)$, respectively, are considered. The carrier frequency is hopped at the start of each packet and the new channel is assumed to have an identically distributed, independent multipath Rayleigh fading. Moreover, partial band interference, which typically exists for FH systems, is not included in the throughput evaluation of nonadaptive FH system. The round-trip delays are the same as those of nonadaptive ARQ schemes.

\section{B. Channel Model}

Mobile radio channel is typically characterized by multipath fading and shadowing. In our simulation, multipath fading is modeled with Rayleigh distribution. Doppler spread induced by the motion of a mobile terminal, which corresponds to the time correlation of the fading gain samples, is also included. Jake's Doppler spectrum is assumed and the correlated Rayleigh fading gain samples are generated by using the method of [11]. The shadowing effect which causes slower variation of the short-term median strength of the received signal is usually modeled with log-normal distribution, i.e., the short-term median strength of the received signal $\gamma(t)$ can be expressed as

$$
\gamma(t)=10^{-\xi(t) / 10}
$$

where $\xi(t)$ is a time-correlated Gaussian random process. In our simulation, the method of [12] is used for log-normal shadowing where the log-normal shadowing is modeled as a Gaussian white noise process which is filtered with first-order low-pass filter. With this model, the discrete-time samples of $\xi(t)$ are given by

$$
\xi_{k+1}=\varepsilon \cdot \xi_{k}+(1-\varepsilon) \cdot \nu_{k}
$$



Fig. 6. Short-term throughput of the adaptive and nonadaptive ARQ schemes for mobile speed of $50 \mathrm{~km} / \mathrm{h}$ and long-term SNR of $10 \mathrm{~dB}$.

where $\nu_{k}$ is a zero-mean white Gaussian random variable with variance $\Omega_{\nu}^{2}, \varepsilon$ is a parameter that controls the spatial correlation of the shadowing and is given by

$$
\varepsilon=\varepsilon_{D}^{v T_{s} / D} \text {. }
$$

The parameter $\varepsilon_{D}$ is the correlation between two points separated by a spatial distance of $D, v$ is the velocity of the mobile terminal, $T_{s}$ is the sampling period. For typical suburban propagation at $900 \mathrm{MHz}$, it has been suggested in [12] that $\Omega_{\xi} \approx 7.5$ $\mathrm{dB}$ with a correlation $\varepsilon_{D} \approx 0.82$ and $D=100$.

\section{Performance of Proposed Adaptive ARQ Scheme}

To illustrate the tracking capability of the proposed adaptive ARQ scheme, the short-term average throughputs are presented in Figs. 6-8 as a function of time for the mobile speed of 50, 10 , and $1 \mathrm{~km} / \mathrm{h}$, respectively. For comparison, the adaptive ARQ scheme with stationary channel assumption [10] and conventional ARQ schemes with single error control codes are also included in these figures. The short-term throughput is obtained by averaging over every 0.1 -s interval.

From the figures, it is observed that for very slow fading case of $v=1 \mathrm{~km} / \mathrm{h}$, the short-term throughput is mainly determined by the multipath fading effect. As the fading rate increases, the effects of shadowing on throughput performance become prominent as can be seen in the cases of $v=10$ and 50 $\mathrm{km} / \mathrm{hr}$.

Regarding the tracking capability to channel conditions, it is observed that both adaptive schemes (the proposed one and the one with stationary channel assumption) can track the channel variations quite well for all fading rates considered, especially for slower fading cases. As expected, both adaptive schemes can adapt to multipath fading more closely for the very slow fading case than for faster fading cases. The tracking trends of both adaptive schemes for faster fading cases cannot follow multipath fading quite closely but they follow the shadowing effect. 


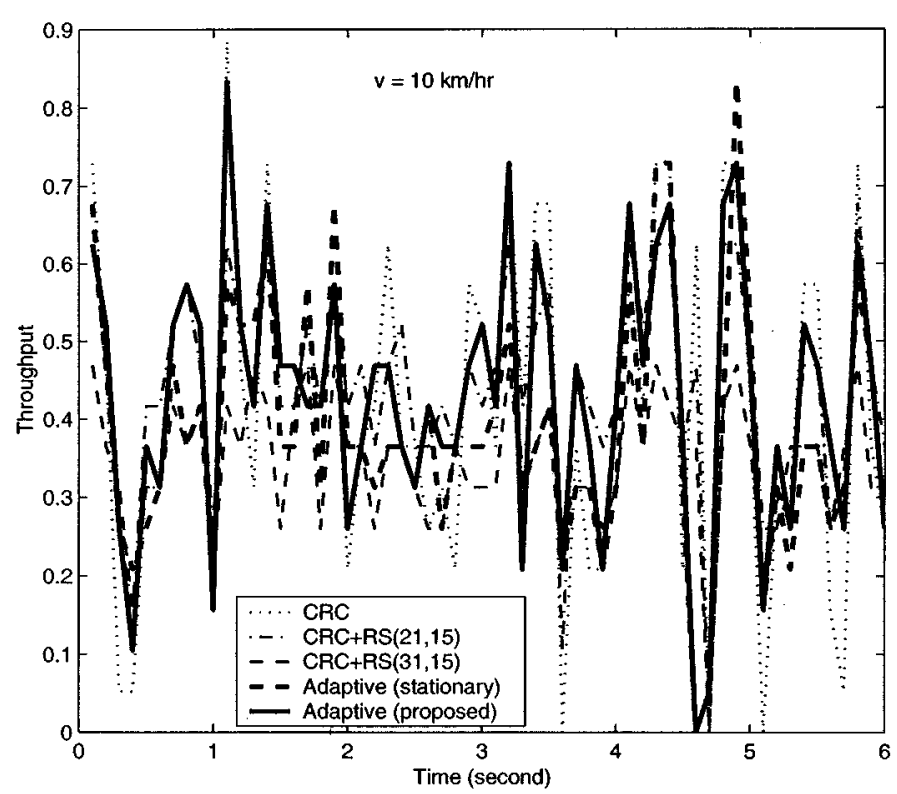

Fig. 7. Short-term throughput of the adaptive and nonadaptive ARQ schemes for mobile speed of $10 \mathrm{~km} / \mathrm{h}$ and long-term SNR of $10 \mathrm{~dB}$.



Fig. 8. Short-term throughput of the adaptive and nonadaptive ARQ schemes for mobile speed of $1 \mathrm{~km} / \mathrm{hr}$ and long term SNR of $10 \mathrm{~dB}$.

For very slow fading rate case of $v=1 \mathrm{~km} / \mathrm{h}$, the channel tracking performances of both adaptive schemes are almost the same as illustrated in Fig. 8. However, as fading rate increases, the channel tracking of the proposed adaptive scheme behaves differently from that of the other scheme. More specifically, for faster fading rates, the proposed adaptive scheme can track the channel variations closer than the other as can be observed in Figs. 6 and 8.

The throughput performances of the adaptive and nonadaptive ARQ schemes as a function of long-term SNR are shown in

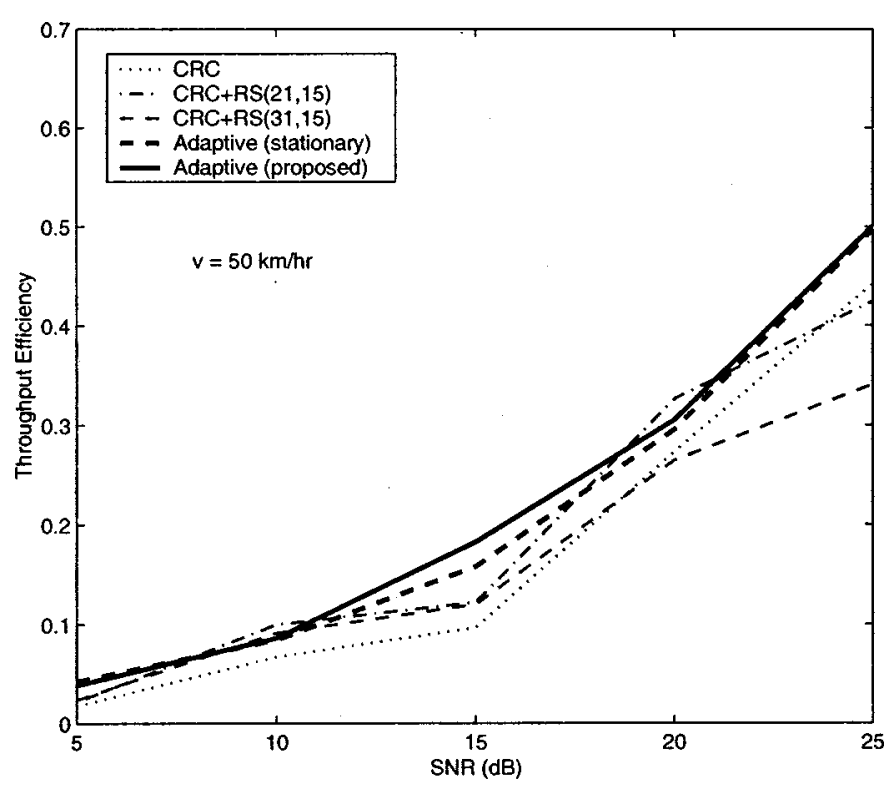

Fig. 9. Throughput comparison of adaptive and nonadaptive ARQ schemes for mobile speed of $50 \mathrm{~km} / \mathrm{h}$.

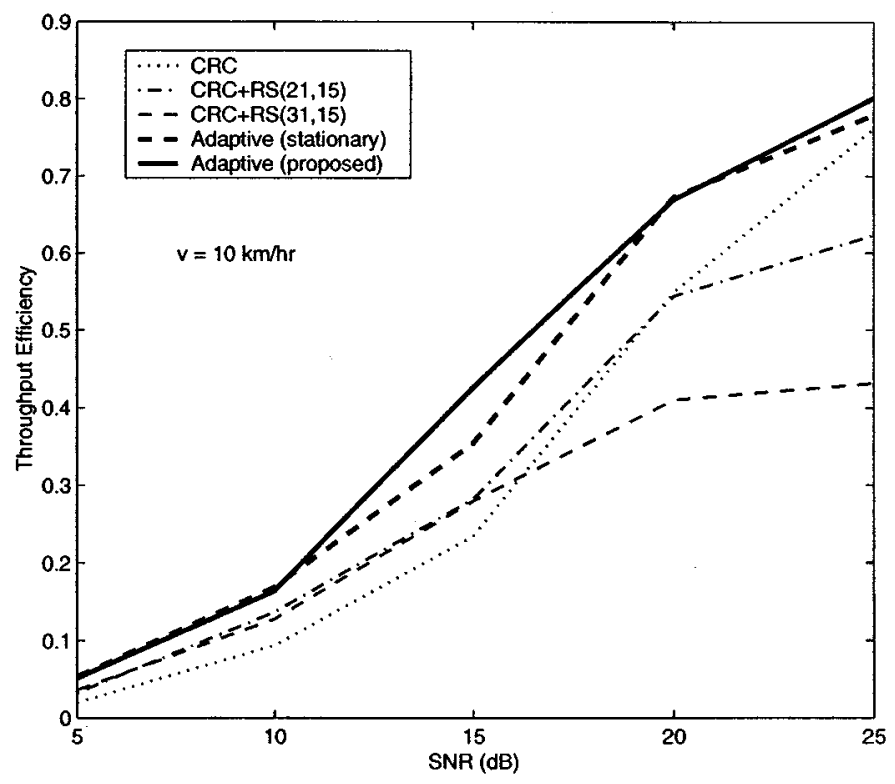

Fig. 10. Throughput comparison of adaptive and nonadaptive ARQ schemes for mobile speed of $10 \mathrm{~km} / \mathrm{h}$.

Figs. 9-11 for the mobile speeds of 50, 10, and $1 \mathrm{~km} / \mathrm{h}$, respectively. Both adaptive schemes show throughput improvement over nonadaptive schemes. For both adaptive schemes, more throughput improvement is observed in slower fading rate cases. This is due to the fact that the adaptive schemes yield better tracking capability over the channel with slower variations. For the cases of mobile speed 1 and $10 \mathrm{~km} / \mathrm{h}$, both adaptive schemes improve throughput with respect to nonadaptive schemes over all SNR values considered.

When comparing two adaptive schemes, at very slow fading case, e.g., $1 \mathrm{~km} / \mathrm{h}$ mobile speed, the proposed scheme has slightly better performance for SNR values greater than $15 \mathrm{~dB}$ but slightly worse performance for SNR values less 


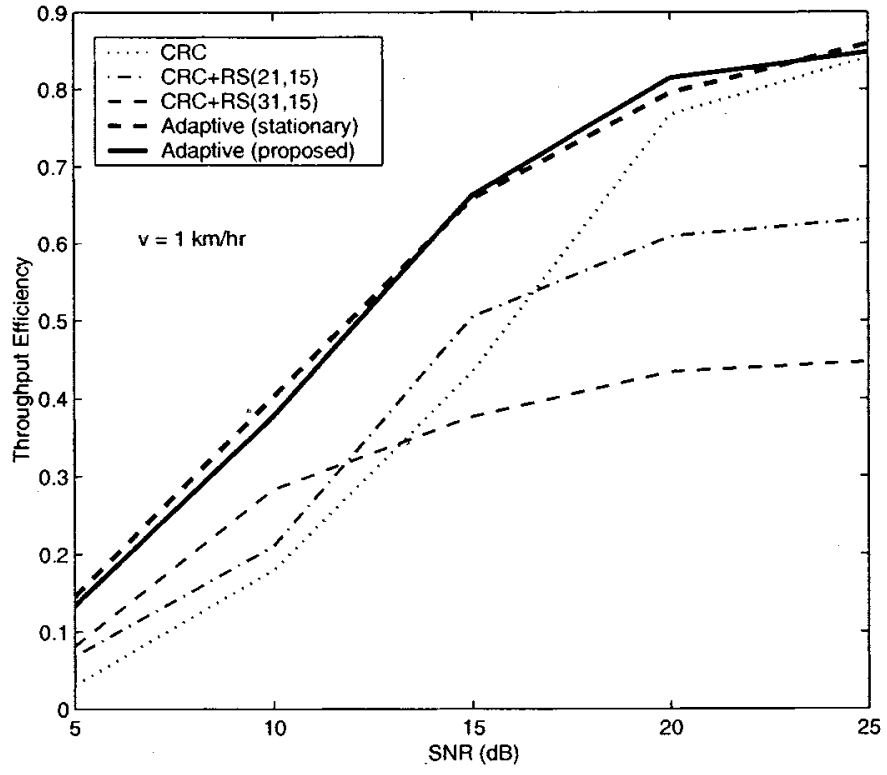

Fig. 11. Throughput comparison of adaptive and nonadaptive ARQ schemes for mobile speed of $1 \mathrm{~km} / \mathrm{h}$.

than $15 \mathrm{~dB}$. As an overall evaluation over all SNR values considered, both schemes perform almost the same for mobile speed of $1 \mathrm{~km} / \mathrm{h}$. In faster fading rate cases of mobile speed 10 and $50 \mathrm{~km} / \mathrm{h}$, the proposed scheme slightly outperforms the adaptive scheme with stationary channel assumption, as an overall. This (slightly) better throughput performance of the proposed scheme indicates the (slightly) better channel tracking capability of the proposed scheme over the other. This fact can also be observed from the short-term throughput figures for faster fading cases. In brief, the proposed adaptive ARQ scheme has a similar performance for slow fading cases and a slightly better performance for fast fading cases than the adaptive ARQ scheme of [10]; but does not require the tedious design task involved in [10].

\section{Performance of Proposed FH-Adaptive ARQ Scheme}

In this subsection, the throughput performances of adaptive ARQ scheme with and without FH are studied. In simulation of an FH-adaptive ARQ scheme, we assume that there is always a new carrier frequency for $\mathrm{FH}$ and the multipath Rayleigh fading channel response for each new carrier frequency is independent and identically distributed (i.i.d.). However, since shadow fading is mainly determined by terrain nature, the packet will face the same shadow fading regardless of carrier frequency. Since FH is applied to counteract deep fades in very slow multipath fading environment, only the case with $1-\mathrm{km} / \mathrm{h}$ mobile speed will be considered.

Fig. 12 shows the throughput performance of the proposed adaptive ARQ scheme without FH and the proposed FH-adaptive ARQ scheme with different values of $\mathrm{FH}$ threshold parameter $\tau_{J u}$. More throughput improvements are observed at lower SNR values. Since more deep fades occur at lower SNR values, the effect of $\mathrm{FH}$ becomes more dominant in throughput efficiency and brings about more throughput gain. A smaller FH threshold parameter value achieves larger throughput improvement since frequency diversity is applied with less

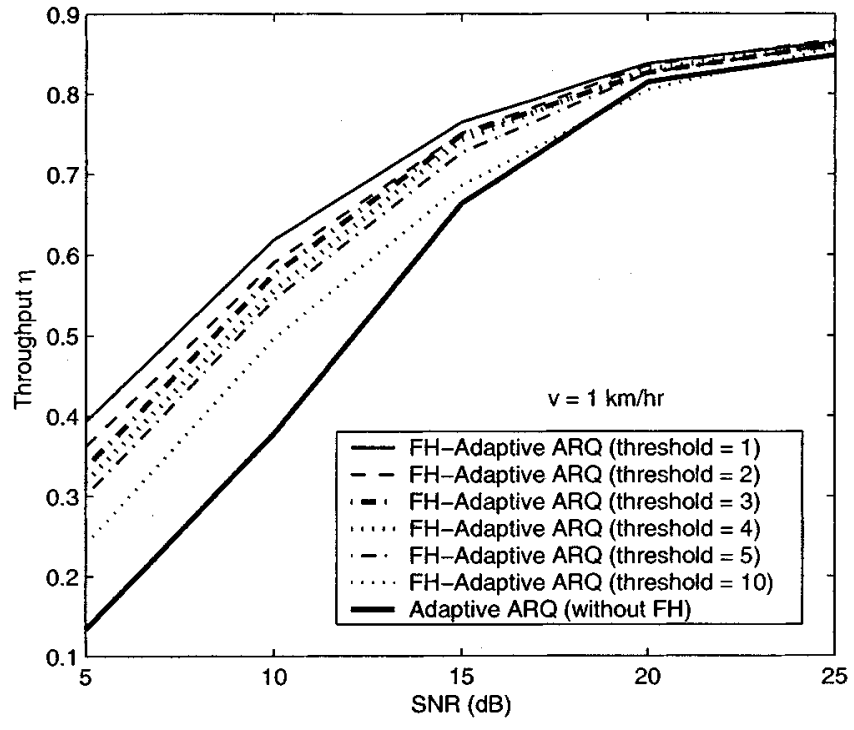

Fig. 12. Throughput comparison of proposed adaptive ARQ schemes with and without FH.



Fig. 13. The average number of FH per successful packet $(\mathrm{FH} \%)$ in FH-adaptive ARQ scheme for different values of FH threshold parameter.

delay. At high SNR values, throughput improvements of FH are almost the same for different values of the FH threshold parameter. Since the probability of deep fade occurrence is very low in a high SNR region, the FH, which is performed when encountering deep fades, has no significant effect on average throughput. Consequently, at high SNR region FH-adaptive ARQ gives almost the same throughput for different values of the FH threshold parameter. Due to the same reason, FH-adaptive ARQ scheme does not achieve significant throughput improvement over adaptive ARQ scheme at high SNR region. However, the throughput improvement achieved by FH-adaptive ARQ is quite significant at long-term SNR region of less than $20 \mathrm{~dB}$ for the system considered.

Fig. 13 shows the corresponding percentages of average number of $\mathrm{FH}$ per successful packet, denoted by $\mathrm{FH} \%$, for different values of $\mathrm{FH}$ threshold parameter. The $\mathrm{FH} \%$ indicates 


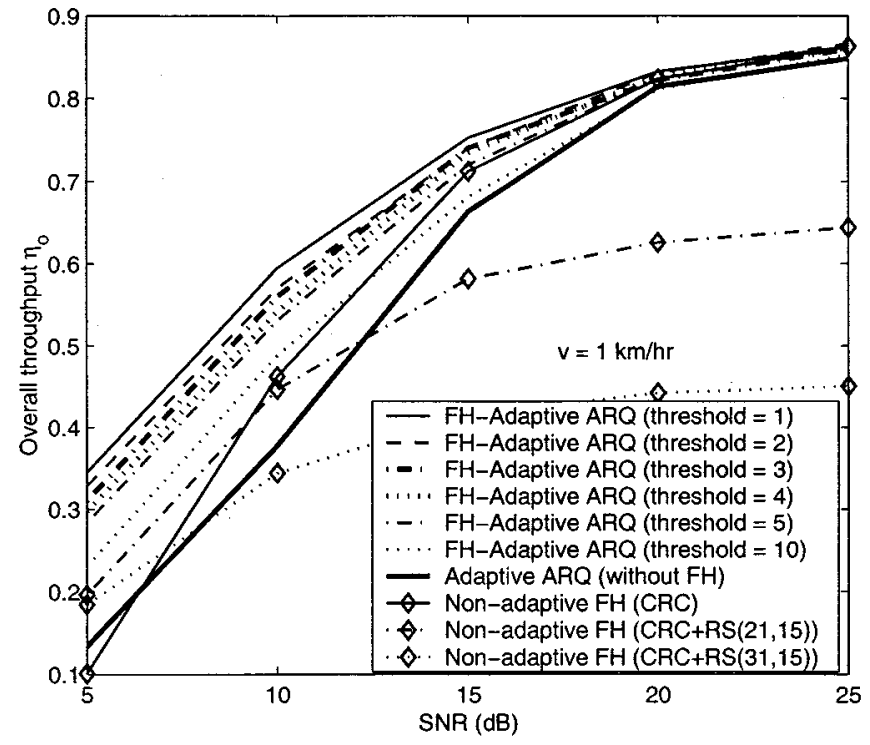

Fig. 14. Overall throughput comparison of proposed adaptive ARQ schemes with and without $\mathrm{FH}$ and nonadaptive $\mathrm{FH}$.

some measure of average signaling load on the system caused by $\mathrm{FH}$, in other words, some measure of system resource usage on control channel by FH. As expected, the average signaling load is quite high at low SNR values for all FH threshold parameter values, with more signaling load for the smaller FH threshold parameter value. The additional resource usage on control channel by FH-adaptive ARQ scheme may require more system resources for control channel. The optimal FH threshold parameter value taking into account the whole system's capacity and throughput would depend on many system parameters and should be evaluated at system level rather than the level and scope considered in this paper.

Taking into account the signaling load of FH setup, the system resource usage of the proposed $\mathrm{FH}$-adaptive ARQ scheme can be evaluated in terms of the overall throughput performance which is defined as the ratio of the number of information bits in a packet to the sum of the average number of bits that would have been transmitted on traffic channel and the average number of bits that would have been transmitted on FH setup control channel for a successful reception of those information bits. Let the FH setup time in terms of the number of bits that would have been transmitted be $R$ and the average throughput on the traffic channel be $\eta$. Then the overall throughput $\eta_{o}$ of the proposed FH-adaptive ARQ scheme can be given by

$$
\eta_{o}=\left(\frac{\mathrm{FH} \% \cdot R}{100 \cdot K}+\frac{1}{\eta}\right)^{-1}
$$

The overall throughputs for different FH threshold parameter values are shown in Fig. 14. By comparing Figs. 12 and 14, it can be seen that although the overall throughputs are less than the traffic channel throughputs and the throughput differences for different $\mathrm{FH}$ threshold parameter values become smaller in Fig. 14, the throughput curves follow a similar trend for different FH threshold parameter values and a smaller FH threshold parameter value still gives better performance. Hence, in terms of system resource usage, FH threshold parameter value of 1 offers the best performance.

Also shown in Fig. 14 are the throughput performances of a random nonadaptive $\mathrm{FH}$ system with different error correction codes. Since no partial band interference is included, the results of the random nonadaptive FH system are quite optimistic. Even compared with those optimistic results of a nonadaptive FH system, the performance of the FH-adaptive ARQ scheme is significantly better. The performance gain of FH-adaptive ARQ over nonadaptive FH with $\mathrm{CRC}$ is negligible at very high SNR values but becomes prominently significant for lower SNR values. It may be explained as follows. At high SNR values, the probability of deep fade occurrence is very low and frequency diversity effect does not have a significant impact on throughput performance; on the other hand, FH-adaptive ARQ would almost always use CRC at high SNR values; hence, both approaches would perform almost the same. For low SNR values, the deep fade occurs more frequently and the noise effect also becomes significant; and, consequently, both frequency diversity and adaptive code diversity have much more impact on throughput performance. Hence, the improvement of having both diversities over having one diversity is greater for lower SNR values. Similarly, the gain of FH-adaptive ARQ over nonadaptive $\mathrm{FH}$ with $\mathrm{CRC}+\mathrm{Reed}-$ Solomon codes can be ascribed to the unnecessary use of a stronger error-correcting code in nonadaptive FH for high SNR values, and having both frequency diversity and adaptive code diversity in FH-adaptive ARQ while having only frequency diversity in nonadaptive FH for low SNR values, respectively.

\section{CONCLUSION}

Adaptive ARQ schemes are quite effective for throughput enhancement in time-varying mobile channel environments. In this paper, we consider ARQ scheme with adaptive error correcting codes, particularly the Reed-Solomon codes. We proposed a channel error rate estimation required for adaptive ARQ implementation by making use of XOR-ing, which is originally applied in [5] and then in [4]. With the proposed channel error rate estimation, we also propose an adaptive scheme that adapts the error correcting code according to channel conditions, as an alternative to [9], [10]. The proposed adaptive ARQ scheme circumvents the tedious throughput calculation and optimization for choosing adaptive system parameters required in [9], [10]. In terms of throughput efficiency, the proposed scheme has similar or slightly better performance than [9], [10].

As an enhancement in counteracting long deep fades, we also investigate the incorporation of the FH concept into the adaptive ARQ scheme. We investigate the impact of the choice of FH threshold parameter value on the throughput performance and the imposed signaling load on the system. Our results show that the FH-adaptive ARQ scheme is quite efficient in counteracting long deep fades at the expense of some signaling load on the system. In terms of system resource usage or overall throughput of the FH-adaptive ARQ scheme, the FH threshold parameter value of 1 gives the best result. The FH-adaptive ARQ also significantly outperforms the random nonadaptive FH system and, hence, it is a promising technique for capacity enhancement. 


\section{REFERENCES}

[1] S. Hara, A. Ogino, M. Araki, M. Okada, and N. Morinaga, "Throughput performance of SAW-ARQ protocol with adaptive packet length in mobile packet data transmission," IEEE Trans. Veh. Technol., vol. 45, pp. 561-569, Aug. 1996.

[2] M. Rice and S. B. Wicker, "Adaptive error control for slowly varying channels," IEEE Trans. Commun., vol. 42, pp. 917-925, Feb./Mar./Apr. 1994.

[3] — "A sequential scheme for adaptive error control over slowly varying channels," IEEE Trans. Commun., vol. 42, pp. 1533-1543, Feb./Mar./Apr. 1994

[4] S. S. Chakraborty, M. Liinaharja, and E. Y-Juuti, "An adaptive ARQ scheme with packet combining for time varying channels," IEEE Commun. Lett., vol. 3, pp. 52-54, Feb. 1999.

[5] P. Sindhu, "Retransmission error control with memory," IEEE Trans. Commun., vol. COM-25, no. 5, pp. 473-479, May 1977.

[6] Y. Yao, "An effective go-back-N ARQ scheme for variable error rate channels," IEEE Trans. Commun., vol. 43, pp. 20-23, Jan. 1995.

[7] A. C. Martins and J. C. Alves, "ARQ protocols with adaptive block size perform better over a wide range of bit error rates," IEEE Trans. Commun., vol. 38, pp. 737-739, June 1990.

[8] S. Kallel and C. Leung, "Analysis of memory and incremental redundancy ARQ schemes over a nonstationary channel," IEEE Trans. Commun., vol. 40, pp. 1474-1480, Sept. 1992.

[9] M. Zeng, H. Minn, and V. K. Bhargava, "An efficient ARQ protocol for adaptive error control over time-varying channel," in ISCOM'99, Kaohsiung, Taiwan, 1999, pp. 72-76.

[10] H. Minn, M. Zeng, A. Annamalai, and V. K. Bhargava, "An efficient ARQ protocol for adaptive error control over time-varying channels," Wireless Personal Commun., vol. 17, no. 1, pp. 3-20, Apr. 2001.

[11] D. J. Young and N. C. Beaulieu, "On the generation of correlated Rayleigh random variates by inverse discrete Fourier transform," in ICUPC' 96 , Cambridge, MA, Sept., pp. 231-235.

[12] M. Gudmundson, "Correlation model for shadow fading in mobile radio systems," Electron. Lett., vol. 27, pp. 2145-2146, Nov. 1991.

[13] S. Lin and D. J. Costello, Error Control Coding: Fundamentals and Applications. Englewood Cliffs, NJ: Prentice-Hall, 1983.

[14] S. B. Wicker and V. K. Bhargava, Reed-Solomon Codes and Their Applications. New York: IEEE Press, 1994.

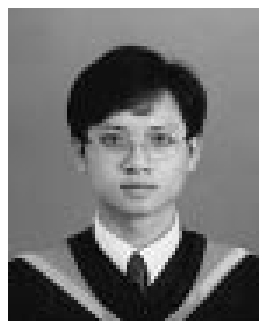

Hlaing Minn (S'97) received the B.E. degree in electronics from Yangon Institute of Technology, Myanmar, in 1995 and the M.Eng. degree in telecommunications from the Asian Institute of Technology (AIT), Thailand, in 1997. Currently, he is working toward the $\mathrm{Ph} . \mathrm{D}$. degree in electrical engineering at the University of Victoria, Victoria, BC, Canada.

He worked as a Laboratory Supervisor at Telecommunications Program, AIT, for one year. Since 1999, he has been a Research Assistant in the Department of Electrical and Computer Engineering at the University of Victoria. His research interests include wireless communications, OFDM, synchronization, channel estimation, modulation, and error-control coding.

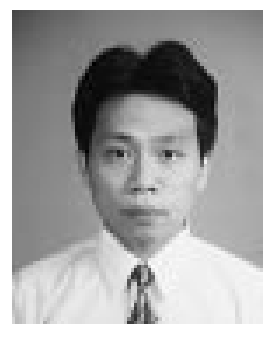

Mao Zeng received the B.Eng., B.Sc., and M.Sc. degrees from Tsinghua University, China, in 1990 and 1993, respectively, and the Ph.D. degree in electrical engineering from the University of Victoria, Victoria, BC, Canada, in 1998.

From May 1998 to December 1999, he was a Postdoctoral Research Fellow at the same institution. In December 1999, he joined Harris Corporation's Advanced Technology Department, Calgary, AB, Canada. He is currently a Senior Staff Engineer at Motorola Inc., Atlanta, GA, and holds an Adjunct Assistant Professor position at the Electrical and Computer Engineering Department of the University of Victoria. His research interests include digital signal processing, error-control coding, and wireless communications.



Vijay K. Bhargava (S'70-M'74-SM'82-F'92) received the B.Sc., M.Sc., and Ph.D. degrees from Queen's University, Kingston, ON, Canada, in 1970, 1972, and 1974 respectively.

Currently, he is Professor of Electrical and Computer Engineering at the University of Victoria, Victoria, BC, Canada. He is a coauthor of the book Digital Communications by Satellite (New York: Wiley, 1981) and co-editor of the IEEE press Book ReedSolomon Codes and Their Applications (1994). His research interests are in multimedia wireless commu-

nications.

Dr. Bhargava is very active in the IEEE and has served as President of the IEEE Information Theory Society (2000); Vice President for the Regional Activities Board (1994, 1995), and Director of Region 7 (1992, 1993). In 1996, he was nominated by the Board of Directors for the Office of IEEE President-Elect. He was Co-Chair for IEEE ISIT' 95, Technical Program Chair for IEEE ICC'99, and is the Chair of IEEE VTC'2002 Fall, to be held in Vancouver, BC, Canada. $\mathrm{He}$ is a Fellow of the BC Advanced Systems Institute, Engineering Institute of Canada (EIC), and the Royal Society of Canada. He is a recipient of the IEEE Centennial Medal (1984), IEEE Canada's McNaughton Gold Medal (1995), the IEEE Haraden Pratt Award (1999), the IEEE Third Millennium Medal (2000), and the IEEE Graduate Teaching Award (2002). 